\title{
Tunable Unidirectional Sound Propagation through a Sonic-Crystal-Based Acoustic Diode
}

\author{
Xue-Feng Li, ${ }^{1} \mathrm{Xu} \mathrm{Ni},{ }^{1}$ Liang Feng, ${ }^{2}$ Ming-Hui Lu,,${ }^{1, *}$ Cheng He, ${ }^{1}$ and Yan-Feng Chen ${ }^{1, \dagger}$ \\ ${ }^{1}$ National Laboratory of Solid State Microstructures and Department of Materials Science and Engineering, Nanjing University, \\ Nanjing 210093, People's Republic of China \\ ${ }^{2}$ Department of Electrical Engineering, California Institute of Technology, Pasadena, California 91125, USA \\ (Received 25 October 2010; revised manuscript received 3 January 2011; published 23 February 2011)
}

Nonreciprocal wave propagation typically requires strong nonlinear materials to break time reversal symmetry. Here, we utilized a sonic-crystal-based acoustic diode that had broken spatial inversion symmetry and experimentally realized sound unidirectional transmission in this acoustic diode. These novel phenomena are attributed to different mode transitions as well as their associated different energy conversion efficiencies among different diffraction orders at two sides of the diode. This nonreciprocal sound transmission could be systematically controlled by simply mechanically rotating the square rods of the sonic crystal. Different from nonreciprocity due to the nonlinear acoustic effect and broken time reversal symmetry, this new model leads to a one-way effect with higher efficiency, broader bandwidth, and much less power consumption, showing promising applications in various sound devices.

DOI: 10.1103/PhysRevLett.106.084301

PACS numbers: 43.35.+d, 42.79.Dj, 43.25.+y

Electrical diodes, due to their capability of rectification of current flux, have significantly revolutionized fundamental science and advanced technology in various aspects of our routine life. Motivated by this one-way effect of electric currents, considerable effort has been dedicated to the study of the unidirectional nonreciprocal transmission of electromagnetic waves, showing important promise in optical and rf communications [1-6]. The realization of such nonreciprocal and unidirectional propagation requires either a broken time reversal symmetry [1-4] or a broken spatial inversion symmetry $[5,6]$ in the artificial photonic structures (e.g., photonic crystals).

Sonic crystals (SCs), in an analogy with the electronic and photonic band structures of semiconductors and photonic crystals, have shown promising impacts in acoustic devices and applications that can efficiently trap, guide, and manipulate sound [7-15]. In the past two decades, with rapid developments in SCs ranging from engineering of band structure for bulk acoustic waves to design of acoustic grating for surface waves, a series of fascinating acoustic effects are consequently demonstrated, such as acoustic band gaps [8,9], negative refractions [10-14], and extraordinary transmission [15]. It is therefore expected, with a sophisticated SC design, that the exotic properties of SCs can lead to more counterintuitive sound manipulation, for example, the realization of acoustic diodes that can break down the conventional transmission reciprocity [16-19]. Similar to electromagnetic wave, sound usually propagates reciprocally back and forth along a given path. Unidirectional flux transmission requires considering the breaking of parity and time symmetry simultaneously in uniform media [20] that do not typically exist in nature. Therefore, SCs are currently considered good candidates to implement nonreciprocal and unidirectional sound propagation. The previous studies proposed the utilization of acoustic nonlinear effects combined with SCs to implement the broken time reversal symmetry as shown in the upper panel of Fig. 1(a) $[16,17]$. The nonlinear medium induces the frequency conversion as the solid red line in Fig. 1(b) indicates, and the adjacent $\mathrm{SC}$ acts as a frequency filter to block the incidence from the right since only the fundamental frequency locates within the band gap. However, the unidirectional transmission from left to right is quite low due to the inherent low conversion efficiency in acoustic nonlinear activities.

In this Letter, we will break the spatial inversion symmetry in the constructed SCs with a sophisticated design approach, and both experimentally and numerically demonstrate unidirectional transmission in such SCs. The constructed SC-based acoustic diodes can be further controlled by simply mechanically manipulating the unit cell of SCs [14] to support either reciprocal or nonreciprocal sound transmission. Our SC-based acoustic diode is a completely linear system without any acoustic nonlinearity. The acoustic diode is designed with an asymmetric periodic

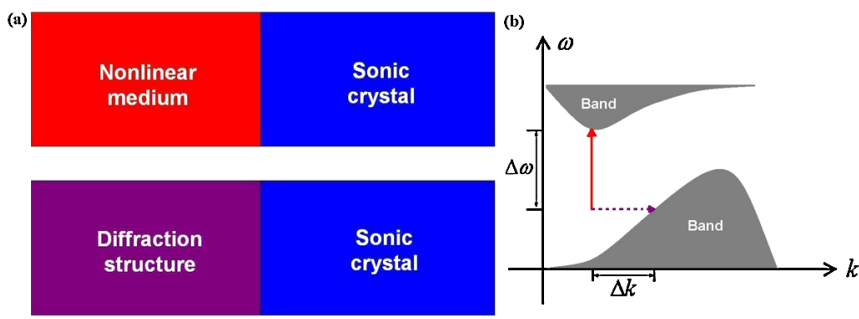

FIG. 1 (color online). (a) Illustration for two diode models, with the top one coupling nonlinear medium to SC and the bottom one using diffraction structure. (b) Schematic for transitions between different modes: the solid red line represents the transition with frequency change and the dashed purple line represents the transition between different spatial modes. 
corrugated SC as shown in the lower panel of Fig. 1(a) that consists of a diffraction structure and a regular SC. The diffraction structure causes the transition between two spatial modes with different spatial frequencies as indicated by the dashed purple line in Fig. 1(b). The adjacent SC thus behaves as a spatial filter due to its intrinsic anisotropy of acoustic band structures, such that different spatial modes, especially high-order diffraction modes with different parallel wave vectors, can be either transferred or prohibited in the designed SC.

The concrete design of the acoustic diode, as shown in Fig. 2(a), consisted of a two-dimensional (2D) SC arranged in a square mesh with a lattice constant of $a=7 \mathrm{~mm}$ and a corrugated diffraction structure with a modulation period of $L=6 a$ in the $y$ direction to the left of the SC. Both the $\mathrm{SC}$ and the diffraction structure were composed of square steel rods with the width of $d=4 \mathrm{~mm}$ in an air background. Finite element simulation was implemented to evaluate the unidirectional transmission property of our acoustic diode, as shown in Fig. 2(d), where the transmission spectra clearly demonstrates nonreciprocal transmission efficiencies for the left incidence (LI) and the right incidence (RI) with a normally incident plane wave. The unidirectional frequency band is indicated by the blue shaded region in Fig. 2(d). In the experiment, the manufactured acoustic diode had 7 corrugation periods in the $y$ direction, and acoustic field scanning measurement was carried out in two ranges of frequencies from 15.0 to
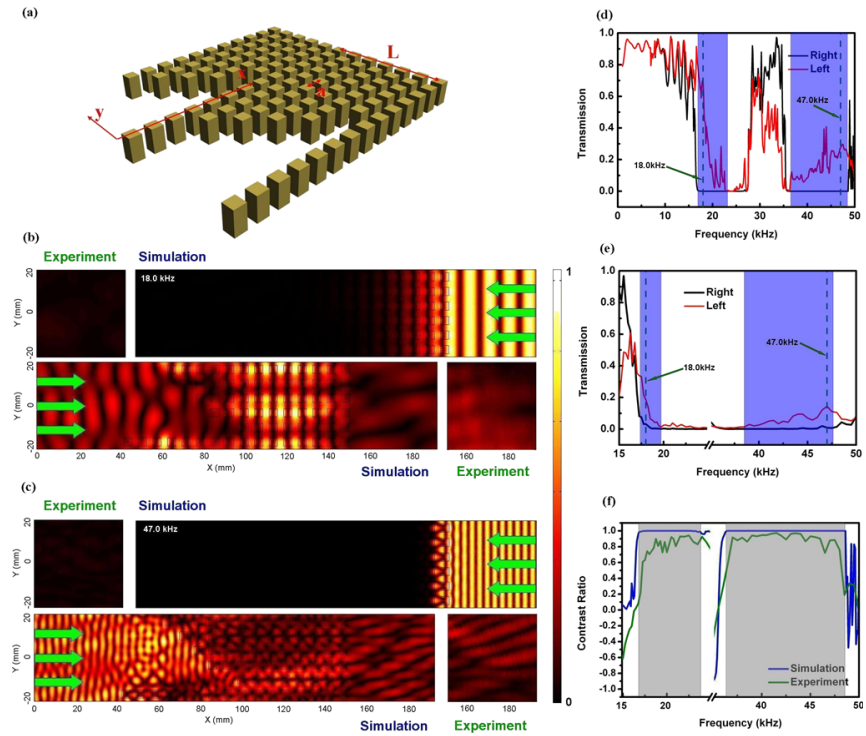

FIG. 2 (color online). (a) Schematic diagram of the SC-based acoustic diode which is periodic in $y$ direction. (b),(c) Simulated and experimental field distribution mappings with the incident wave frequency at 18.0 and $47.0 \mathrm{kHz}$, respectively. Green arrows indicate the propagation directions. (d),(e) Numerically calculated and experimentally measured transmission spectra of LI and RI, respectively. Green arrows indicate the frequency at which field distribution is mapped. (f) Contrast transmission ratio of the acoustic diode.
$25.0 \mathrm{kHz}$ and from 35.0 to $50.0 \mathrm{kHz}$, due to the limited response range of transducers. In spite of limitations of the finite size in the transverse direction and slight imperfection of the plane wave source in the experiment, the measurement results shown in Fig. 2(e) still agreed with the simulation. Especially within $17.5-19.5 \mathrm{kHz}$ and $38.7-$ $47.5 \mathrm{kHz}, \mathrm{LI}$ was associated with high transmission efficiency, but transmission was not allowed for RI, showing a relatively broadband unidirectional transmission only for LI as indicated by the blue shaded region in Fig. 2(e). In order to illustrate this unidirectional transmission phenomenon more clearly, spatial intensity distributions of the acoustic pressure field are mapped out both numerically and experimentally as shown in Figs. 2(b) and 2(c) at two frequencies of 18.0 and $47.0 \mathrm{kHz}$, respectively. Because of the SC's directional band gap in the $x$ direction $(\Gamma-X)$, RI was almost completely reflected without any transmission. In the case of LI, however, strong acoustic field can be observed in the output area; for example, the transmission at $18.0 \mathrm{kHz}$ was about $69 \%$. But since the outgoing waves are not parallel to the incident waves, it is evident that energy in the normal incidence was converted, through high-order diffractions, to other spatial modes that have different spatial frequencies to overcome the barrier imposed by the $\Gamma-X$ directional band gap. The unidirectional transmission was therefore established and the output field was actually the interference of outgoing beams from different diffraction orders.

To clearly quantify the performance of the acoustic diode, we defined the contrast ratio $\left(R_{c}\right)$ as

$$
R_{c}=\frac{T_{L}-T_{R}}{T_{L}+T_{R}}
$$

where $T_{L}$ and $T_{R}$ are the transmissions for LI and RI, respectively. The absolute value of $R_{c}$ represents the relative transmission weight between these two incident cases. $R_{c}$ was also evaluated as a function of frequency as shown in Fig. 2(f). Within the specific frequency ranges where unidirectional transmission was clearly observed, $R_{c}$ reaches 1 with a good agreement with experiments and simulations, evidently manifesting great performance of our acoustic diode.

In addition to the broken spatial symmetry resulting from the diffraction structure, the building block of our $\mathrm{SC}$, the steel square rod also introduces a broken rotational symmetry to the unit cell itself [14]. The introduced broken rotational symmetry can cause the change of the SC's band dispersion by simply mechanically rotating all the square rods with the same lattice configuration, as different rotations affect the effective scattering section for acoustic waves significantly. It is therefore expected to effectively control the sound rectification (i.e., efficiently tune the relative transmission weight between LI and RI) with our acoustic diode by rotating the rods. In the experiment, we rotated all the square rods $45^{\circ}$ with keeping other parameters the same in the diode configuration, as shown 
in Fig. 3(a). Intrinsic characteristics of multiple scatterings in the SC were consequently dramatically changed, leading to a broader first band gap of SC as shown in Fig. 3(d) due to the increase of the effective scattering section. The unidirectional transmission could still be observed from 16.2 to $22.3 \mathrm{kHz}$. But from 40.0 to $50.0 \mathrm{kHz}$, transmission was allowed for both LI and RI and the one-way phenomenon was deactivated. Therefore, it is evident that the sound rectification effect could be effectively turned on or off in our acoustic diode by rotating the square rods of the SC. This tunable acoustic diode was further confirmed with mappings of spatial intensity distribution of the acoustic pressure field at 17.25 and $47.0 \mathrm{kHz}$, as shown in Figs. 3(b) and 3(c), respectively. Consistent with transmission spectra in Figs. 3(d) and 3(e), pronounced unidirectional transmission can be seen at $17.25 \mathrm{kHz}$, but equivalent transmission was obtained in both directions at $47.0 \mathrm{kHz}$. The contrast ratio in Fig. 3(f) also confirmed that the relative transmission weight is 1 from 16.5 to $22.5 \mathrm{kHz}$, and the unidirectional character thus remains the same (compared to Fig. 2), while the weight was around 0 from 35.0 to $50.0 \mathrm{kHz}$, showing the change from unidirectional to bidirectional transmission. In addition, this tunable unidirectional transmission effect could also be influenced by different rotation angles and filling fractions (see Fig. S4 in [21]). In principle, with a more systematic design of the square-rod-based SC, this tunable acoustic diode could be constructed either in the first or the second band (as demonstrated above), or both. And the frequency range
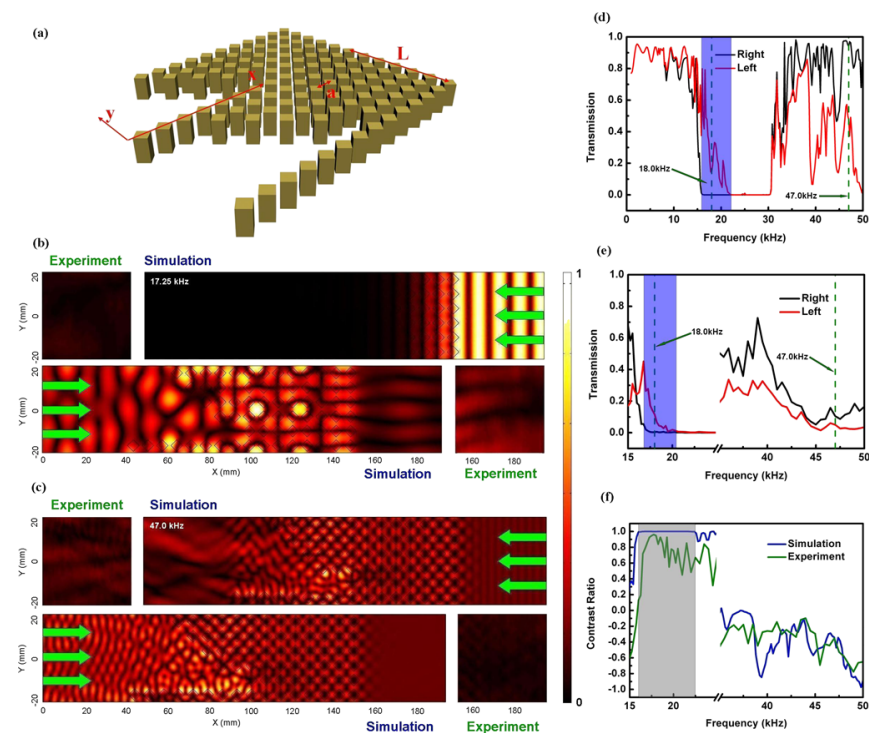

FIG. 3 (color online). (a) Schematic diagram of the SC-based acoustic diode after rotating the square rods. (b),(c) Simulated and experimental field distribution mappings with the incident wave frequency at 17.25 and $47.0 \mathrm{kHz}$, respectively. Green arrows indicate the propagation directions. (d),(e) Numerically calculated and experimentally measured transmission spectra of LI and RI, respectively. Green arrows indicate the frequency at which field distribution is mapped. (f) Contrast transmission ratio of the acoustic diode. of unidirectional transmission might be anticipated to be effectively controlled with different rotational angles of these square rods.

To gain deeper insight, we analytically investigated the mechanism underlying this unidirectional transmission phenomenon. With a plane wave incidence the acoustic pressure field in the input and output half-spaces can be Fourier expanded as

$$
\begin{gathered}
p(x, y)=\exp \left(j k_{0} x\right)+\sum_{n=-\infty}^{\infty} \rho_{n} \exp \left(j \alpha_{n} y+j \beta_{n} x\right), \\
p(x, y)=\sum_{n=-\infty}^{\infty} \tau_{n} \exp \left(j \alpha_{n} y+j \beta_{n} x\right),
\end{gathered}
$$

respectively, where $\alpha_{n}$ and $\beta_{n}$ are wave numbers in the $x$ and $y$ directions, respectively, and $\beta_{n}=\sqrt{k_{0}^{2}-\alpha_{n}^{2}}, \alpha_{n}=$ $2 n \pi / L$ ( $n$ is the diffraction order), $k_{0}$ is the free-space wave number, and $\rho_{n}$ and $\tau_{n}$ are amplitudes of the $n$ thorder diffraction beams in reflection and transmission, respectively. The mechanism of our acoustic diode is closely relevant to these two diffraction terms. These diffraction terms represent the energy conversion among different diffraction orders. The difference of structure geometries on two sides resulted in different diffraction orders, and only the orders locating within the propagating band of the SC could transmit the energy from one side to the other. Notice that, however, the diode in our study cannot be completely analytically described by Eqs. (2) and (3), due to its complex diffraction construction. Therefore, simplified diode geometry was studied and compared [21]. As shown in Figs. S2(b) and S2(d) of [21], tunable unidirectional transmission effect is also realized with a much weaker diode behavior. The optimized complex geometry in this Letter has advantages over this simplified diode in two aspects. (1) With the adiabatic change in the complex asymmetric corrugation, the reflection of the acoustic wave incident from the left side could be efficiently reduced. (2) Compared to diffractions through the simpler corrugation, it is more efficient to generate higher order diffraction modes through the complex asymmetric corrugation due to the large slope angle.

In order to illustrate nonreciprocal sound propagation in the acoustic diode, we further analyze the equifrequency surfaces (EFSs) at different frequencies of the $\mathrm{SC}$ as shown in Fig. 4. For the case of RI in Figs. 4(a) and 4(b), high diffraction orders completely fell in the evanescent regime ( $\rho_{n}$ and $\tau_{n}$ are 0 ) in the interested frequency ranges, and thus all the energy was still conserved in the zero order, i.e., normal incidence to the SC, which was located within the directional band gap of the SC. Therefore, sound transmission for RI was prohibited by the SC. However, for the case of LI, the existence of the diffraction structure before the SC converted the normal incidence mode to high-order diffraction modes. This can be more clearly visualized in Fig. S3 of [21] where an incident beam with a finite width 


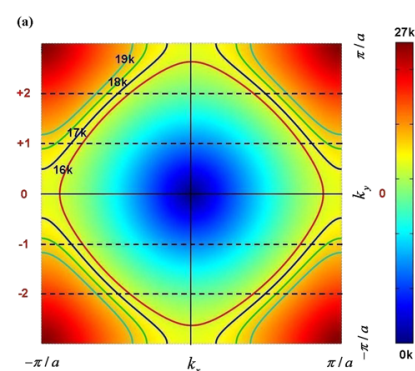

$$
\text { (c) }
$$
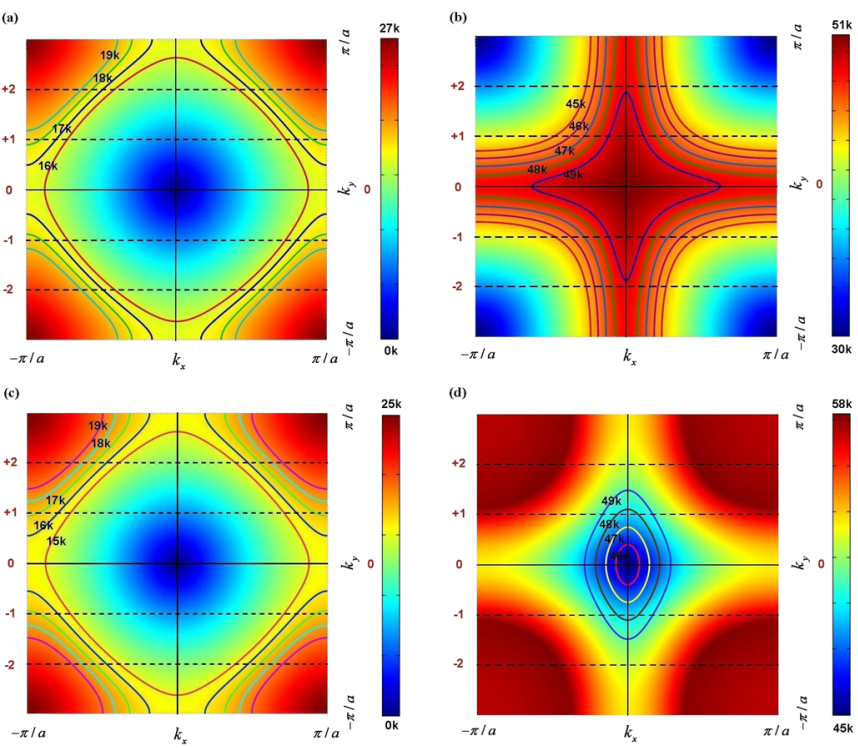

FIG. 4 (color online). (a),(b) EFSs of the SC in first Brillouin zone from 16 to $19 \mathrm{kHz}$ and from 45 to $49 \mathrm{kHz}$, respectively. The numbers $(-2,-1,0,+1,+2)$ on left and right sides correspond to the diffraction orders. (c),(d) EFSs of the SC after the rotation of rods in two frequency ranges.

was considered. The transmitted beams propagated along different directions, corresponding to different positive and negative diffraction orders due to the complex diffraction structure. Consistent with EFSs in Figs. 4(a) and 4(b), \pm 1 and \pm 2 modes significantly contributed to transmission through the SC and resulted in unidirectional sound transmission. After the rotation of the square rods, EFS from 17 to $19 \mathrm{kHz}$ remained almost the same as shown in Fig. 4(c), demonstrating a nonreciprocal feature as all the diffracted beams easily transmit through the SC for LI as shown in Fig. S3(f) of [21]. But from 45 to $49 \mathrm{kHz}$, EFS was drastically changed as shown in Fig. 4(d), and the original directional band gap in Fig. 4(b) disappeared. Therefore, sound transmission was also allowed for RI, and the nonreciprocal sound propagation is thus expected to be simply mechanically controlled by rotating the rods of our acoustic diode.

To summarize, we have theoretically proposed and experimentally constructed a sonic-crystal-based acoustic diode with a broken spatial inversion symmetry in which nonreciprocal propagation and unidirectional transmission of sound were clearly observed. Furthermore, the introduced broken rotational symmetry of the unit cell of the sonic crystal results in a sophisticated mechanical manipulation of the observed nonreciprocal sound transmission. Compared to previously proposed acoustic diodes using nonlinearities, our system is a completely linear system, showing advantages such as broadband operation, high conversion efficiency, and much less power consumption. The same concept is also expected to implement an on-chip isolator for various types of acoustic waves, such as surface acoustic waves [22] and Lamb waves [23].

The work was jointly supported by the National Basic Research Program of China (Grant No. 2007CB613202). We also acknowledge the support from the Nature Science Foundation of China (Grant No. 10874080) and the Nature Science Foundation of Jiangsu Province (Grant No. BK2007712).

*To whom correspondence should be addressed. luminghui@nju.edu.cn

${ }^{\dagger}$ To whom correspondence should be addressed. yfchen@nju.edu.cn

[1] Z. Wang et al., Phys. Rev. Lett. 100, 013905 (2008).

[2] F. D. M. Haldane and S. Raghu, Phys. Rev. Lett. 100, 013904 (2008).

[3] C. He et al., Appl. Phys. Lett. 96, 111111 (2010).

[4] Z. Yu and S. Fan, Nat. Photon. 3, 91 (2009).

[5] A. E. Serebryannikov, Phys. Rev. B 80, 155117 (2009).

[6] A.E. Serebryannikov and E. Ozbay, Opt. Express 17, 13335 (2009).

[7] M. H. Lu, L. Feng, and Y.F. Chen, Mater. Today 12, 34 (2009)

[8] M. S. Kushwaha et al., Phys. Rev. Lett. 71, 2022 (1993).

[9] R. Martínez-Sala et al., Nature (London) 378, 241 (1995).

[10] M. H. Lu et al., Nature Mater. 6, 744 (2007).

[11] L. Feng et al., Phys. Rev. Lett. 96, 014301 (2006).

[12] L. Feng et al., Phys. Rev. B 72, 033108 (2005).

[13] X.D. Zhang and Z. Y. Liu, Appl. Phys. Lett. 85, 341 (2004).

[14] L. Feng et al., Phys. Rev. B 73, 193101 (2006).

[15] Y. Zhou et al., Phys. Rev. Lett. 104, 164301 (2010).

[16] B. Liang, B. Yuan, and J. C. Cheng, Phys. Rev. Lett. 103, 104301 (2009).

[17] B. Liang et al., Nature Mater. 9, 989 (2010).

[18] B. W. Li, L. Wang, and G. Casati, Phys. Rev. Lett. 93, 184301 (2004).

[19] V. F. Nesterenko et al., Phys. Rev. Lett. 95, 158702 (2005).

[20] C. E. Ruter et al., Nature Phys. 6, 192 (2010).

[21] See supplemental material at http://link.aps.org/ supplemental/10.1103/PhysRevLett.106.084301 for detailed elucidation of the contribution of different diffraction modes to the one-way effect and influence of rotation angles and filling fraction on the tunability.

[22] J.H. Sun and T.T. Wu, Phys. Rev. B 74, 174305 (2006).

[23] C. Y. Huang, J. H. Sun, and T. T. Wu, Appl. Phys. Lett. 97, 031913 (2010). 